\title{
Chaotic jam and phase transition in traffic flow with passing
}

\author{
Takashi Nagatani \\ Division of Thermal Science, College of Engineering, Shizuoka University, Hamamatsu 432-8561, Japan
}

(Received 19 October 1998)

\begin{abstract}
The lattice hydrodynamic model is presented to take into account the passing effect in one-dimensional traffic flow. When the passing constant $\gamma$ is small, the conventional jamming transition occurs between the uniform traffic and kink density wave flows. When passing constant $\gamma$ is larger than the critical value, the jamming transitions occur from the uniform traffic flow, through the chaotic density wave flow, to the kink density wave flow, with an increasing delay time. The chaotic region increases with passing constant $\gamma$. The neutral stability line is derived from the linear stability analysis. The neutral stability line coincides with the transition line between the uniform traffic and density wave flows. The modified Korteweg-de Vries equation describing the kink jam is derived for small values of $\gamma$ by use of a nonlinear analysis.
\end{abstract}

[S1063-651X(99)15308-X]

PACS number(s): 05.70.Fh, 05.70.Jk, 89.40.+k

\section{INTRODUCTION}

Recently, traffic problems have attracted considerable attention [1-6]. The jamming transitions between freely moving traffic and jammed traffic have been found in the traffic models. In the freely moving traffic, the car density is low and distributed uniformly on a highway. In the jammed traffic, the density wave of high density propagates backward into the low-density flow. The transitions are very similar to the conventional phase transitions and critical phenomena [7-15].

The jamming transition has been described in terms of the thermodynamic terminology of phase transitions and critical phenomena $[16,17]$. The freely moving traffic and jammed traffic correspond, respectively, to the gas and liquid phases in the conventional gas-liquid phase transition: the car density or headway corresponds to the density or volume (the order parameter) and the sensitivity parameter (the inverse of the delay time) corresponds to temperature. The coexisting curve, spinodal line, and critical point are obtained from the derivatives of the thermodynamic potential that is derived from the traffic models $[16,17]$.

The density wave in the coexisting phase appears with the kink-antikink form in the car following models that are not stochastic but deterministic $[3,14,15]$. Once the density wave is formed, it is robust and regular. The kink density wave neither breaks up nor coalesces with others in the steady state, except for the early stage. To our knowledge, the appearance of a chaotic traffic jam has not yet been reported. The chaotic traffic jam means that the density waves (traffic jams) become unstable, break up, and coalesce irregularly in the deterministic models. There is an important question as to whether or not the chaotic jam occurs in the deterministic traffic models. Does the density wave break up and coalesce with others in the chaotic traffic flow? Does the passing have an important effect on the traffic flow?

In this paper, we extend the single-lane traffic model (described by the lattice hydrodynamic equations) to take into account the passing effect. We investigate the effect of passing on the traffic flow. We would like to address whether or not the traffic flow exhibits the chaotic behavior by introduc- ing the passing effect into the deterministic model. We show that the chaotic jam indeed appears in the deterministic traffic model. Taking the passing into account makes the phase diagram much richer. Besides the known jammed and nonjammed phases, a new phase (chaotic jam) is identified. We apply the linear analysis to the traffic model. We show that the neutral stability line coincides with the phase boundary line between the uniform traffic flow without jams and the density wave phase. Furthermore, we prove that the density wave can be described by the modified Korteweg-de Vries $(\mathrm{KdV})$ equation for small passing constant.

\section{LATTICE MODEL}

For later convenience, we present the lattice version of the continuum model on a single-lane highway without passing [17]. The lattice hydrodynamic model is described by the following difference-difference equation with time lag $\tau$.

$$
\begin{gathered}
\rho_{j}(t+\tau)-\rho_{j}(t)+\tau \rho_{0}\left[\rho_{j}(t) \mathrm{\vee}_{j}(t)-\rho_{j-1}(t) \mathrm{v}_{j-1}(t)\right]=0, \\
\rho_{j}(t+\tau) \mathrm{v}_{j}(t+\tau)=\rho_{0} V\left(\rho_{j+1}(t)\right),
\end{gathered}
$$

where the subscript $j$ indicates site $j$ on the one-dimensional lattice. $\rho_{j}(t)$ and $\mathbf{v}_{j}(t)$ represent, respectively, the density and velocity on site $j$ at time $t . \rho_{0}$ is the average density. Equation (1) is the lattice version of the continuity equation that relates local density $\rho_{j}(t)$ to local average speed $\mathrm{v}_{j}(t)$. Space variable $j$ is a dimensionless variable divided by average headway $1 / \rho_{0}$. The lattice spacing is taken to be average headway $1 / \rho_{0}$. Equation (2) is the evolution equation in place of the Navier-Stokes equation. The function $V\left(\rho_{j}(t)\right)$ is called as the optimal velocity. It is given by

$$
V\left(\rho_{j}(t)\right)=\tanh \left(\frac{2}{\rho_{0}}-\frac{\rho_{j}(t)}{\rho_{0}^{2}}-\frac{1}{\rho_{c}}\right)+\tanh \left(\frac{1}{\rho_{c}}\right) .
$$

This function has the turning point (inflection point) at $\rho_{j}(t)=\rho_{c}$ when $\rho_{0}=\rho_{c}$, where $\rho_{0}$ is the average density and $\rho_{c}$ is the inverse of the safety distance $[3,16,17]$. We introduce the parameter $\rho_{0}$ to be $-\rho_{0}^{2} V\left(\rho_{0}\right)^{\prime}=1$ when $\rho_{0}=\rho_{c}$. 
Generally, it is necessary that the optimal velocity function have the following properties: being a monotonically decreasing function, having an upper bound (maximal velocity), and having a turning point at the safety distance [17]. It is important that the optimal velocity function have a turning point. Otherwise, one cannot obtain a kink-antikink density wave representing the stable traffic jam.

The delay time $\tau$ allows for the time lag that it takes the traffic current to reach the optimal current $\rho_{0} V\left(\rho_{j+1}(t)\right)$ when the traffic flow is varying. The idea is that traffic current $\rho_{j}(t) \mathrm{V}_{j}(t)$ on site $j$ at time $t$ is adjusted by the optimal current $\rho_{0} V\left(\rho_{j+1}(t-\tau)\right)$ on site $j+1$ at time $t-\tau$. This is similar to the idea of the car-following model analyzed by Newell [18] and Whitham [19]. We have performed the computer simulation for the above model and confirmed that the kink density wave of the jam occurs when the density is larger than the critical value. We have also analyzed the above model and derived the modified $\mathrm{KdV}$ equation, and time-dependent Ginzburg-Landau (TDGL) equation [17].

We extend the above model to take into account the passing. The continuity equation (1) does not change by the passing but the evolution equation (2) changes. When the traffic current on site $j$ is larger than that on site $j+1$, a passing occurs. We assume that the traffic quantity of the passing on site $j$ is proportional to the difference between the optimal traffic currents on sites $j$ and $j+1$. Then, the evolution equation taking account of the passing is given by

$$
\begin{aligned}
\rho_{j}(t+\tau) \mathrm{\vee}_{j}(t+\tau)= & \rho_{0} V\left(\rho_{j+1}(t)\right)+\gamma\left[\rho_{0} V\left(\rho_{j+1}(t)\right)\right. \\
& \left.-\rho_{0} V\left(\rho_{j+2}(t)\right)\right],
\end{aligned}
$$

where $\gamma$ is the passing constant. $\rho_{0} V\left(\rho_{j+1}(t)\right)$ is the optimal traffic current on site $j+1$ at time $t$ and $\rho_{0} V\left(\rho_{j+2}(t)\right)$ is the optimal current on site $j+2$ at time $t$. The traffic flow with the passing is described by Eqs. (1) and (4) with Eq. (3).

By eliminating the velocity in Eqs. (1) and (4), one obtains the density equation

$$
\begin{aligned}
& \rho_{j}(t+2 \tau)-\rho_{j}(t+\tau)+\tau \rho_{0}^{2}\left[V\left(\rho_{j+1}(t)\right)-V\left(\rho_{j}(t)\right)\right] \\
& \quad-\gamma \tau \rho_{0}^{2}\left[V\left(\rho_{j+2}(t)\right)-2 V\left(\rho_{j+1}(t)\right)+V\left(\rho_{j}(t)\right)\right]=0 .
\end{aligned}
$$

The last term on the left-hand side of Eq. (5) represents the density variation caused by the passing. When $\gamma=0$., Eq. (5) reproduces the density equation without the passing. It will be expected that the traffic behavior changes with increasing passing constant $\gamma$.

\section{SIMULATION}

We carry out a computer simulation for the traffic-flow model described by Eq. (5). We discretize time $t$ by time interval $\tau$. Now, the symbol $t$ represents the time step number. Then, from Eq. (5), one obtains

$$
\begin{aligned}
& \rho_{j}(t+2)-\rho_{j}(t+1)+\tau \rho_{0}^{2}\left[V\left(\rho_{j+1}(t)\right)-V\left(\rho_{j}(t)\right)\right] \\
& \quad-\gamma \tau \rho_{0}^{2}\left[V\left(\rho_{j+2}(t)\right)-2 V\left(\rho_{j+1}(t)\right)+V\left(\rho_{j}(t)\right)\right]=0 .
\end{aligned}
$$

Since $\tau, \rho_{0}$, and $V$ have dimensions of [time], [length $]^{-1}$, and [length]/[time], respectively, the second and third terms have the dimension of density. This is consistent with the dimension of the first term. Parameter $\tau$ is the time interval in the difference equation (6) and is important in the traffic model.

We consider the nonrandom initial condition in order to study the chaotic behavior in the deterministic model. Initially, the density is assumed to be a step function such as

$\rho_{j}(0)= \begin{cases}\rho_{0}-A & \text { for } 0 \leqslant j<\frac{L}{2}, \\ \rho_{0}+A & \text { for } \frac{L}{2} \leqslant j<L,\end{cases}$

$\rho_{j}(1)= \begin{cases}\rho_{0}-A & \text { for } 0 \leqslant j<\frac{L}{2}-m, \quad L-m \leqslant j L, \\ \rho_{0}+A & \text { for } \frac{L}{2}-m \leqslant j<L-m,\end{cases}$

where $A$ is a constant, $m$ is a positive integer, and $L$ is the system size. The boundary is periodic. We performed the simulation for various values of average density $\rho_{0}$ and delay time $\tau$ for $0 \leqslant \gamma \leqslant 0.4$, where $\rho_{c}=0.2$ and $100 \leqslant L \leqslant 400$. As a result, the four types of traffic flow are distinguished: (i) a uniform traffic flow with low density, (ii) a traffic flow with kink density waves, (iii) a traffic flow with chaotic density waves, and (iv) a uniform traffic flow with high density. Besides the known traffic flows (i), (ii), and (iv), a new traffic flow (iii) is identified. In what follows, we will justify the term "chaotic" for the new traffic flow (iii) by computing Fourier spectra, Lyapunov exponents, and phase-space plots. Taking passing into account makes the new phase. Typical patterns of two traffic flows (ii) and (iii) with density waves are shown in Fig. 1. Part (a) shows the space-time evolution of density for $\gamma=0.3, a(=1 / \tau)=3.5$, and $\rho_{0}=0.2$ where $L$ $=100$ and $t=20000-20200$. It is a typical traffic pattern of type (ii). It exhibits a kink-antikink density wave. The kink density wave is stable and robust, except for the early stage. The kink density wave is similar to that observed in the traffic flow with no passing. Part (b) shows the space-time evolution of density for $\gamma=0.3, a(=1 / \tau)=5.0$, and $\rho_{0}$ $=0.2$ where $L=100$ and $t=20000-20200$. It is a typical traffic pattern of type (iii). It exhibits irregular density waves. The density waves coalesce with one another (seen in $a$ $\rightarrow b$ ) and break up (seen in $c \rightarrow d$ ). The chaotic traffic flow is induced by introducing the passing effect into the original single-lane traffic model [17]. Both density waves in Figs. 1(a) and 1(b) propagate backward and represent the traffic jams.

In order to justify the term "chaotic," we compute the Fourier spectra, the phase-space plots, and the Lyapunov exponents. Figure 2 shows the Fourier spectra of time series of density where $t=30000-40000$. The Fourier spectra correspond to the traffic patterns (a) and (b) in Fig. 1, respectively. The kink density wave (a) exhibits the typical structure of a periodic function. The chaotic density waves exhibit the typical structure of a nonperiodic function. The traffic flows (i), (ii), and (iv) are the typical traffic patterns appearing for traffic with no passing. The chaotic density waves (iii) appear for traffic with passing.

We study the phase diagram for the traffic flow with passing. Figure 3 shows the phase diagram in parameter space 


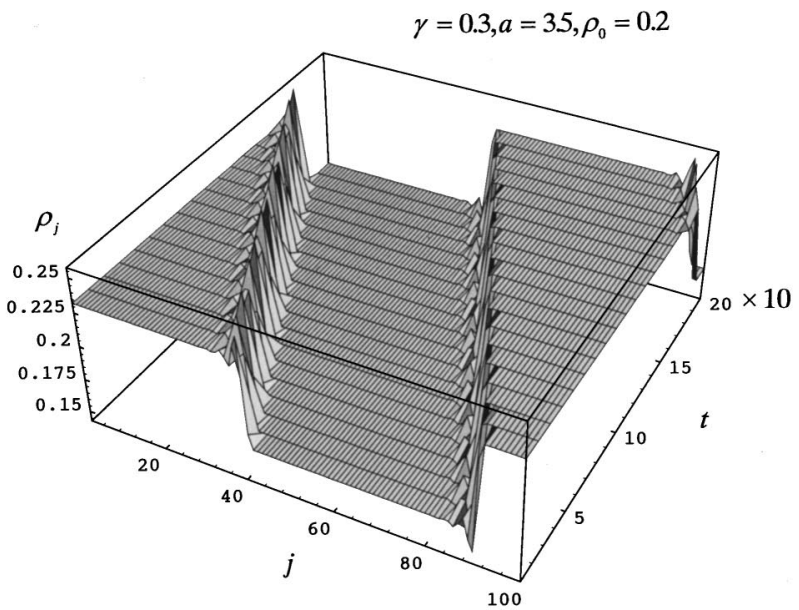

(a)

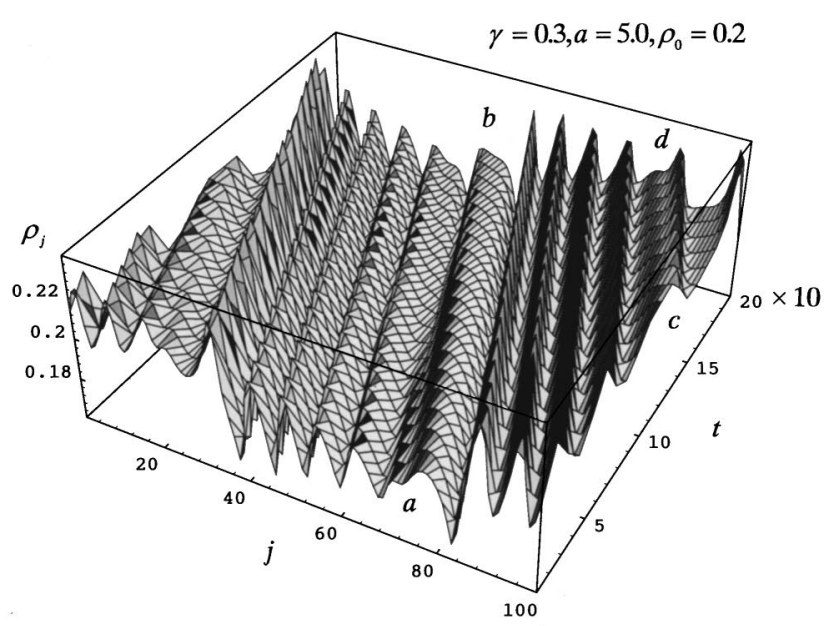

(b)

FIG. 1. Two typical patterns of the space $(j)$-time $(t)$ evolution of density $\rho_{j}$ for passing constant $\gamma=0.3$ and average density $\rho_{0}$ $=0.2$ between $t=20000$ and 20200 where system size $L$ is 100 . (a) The kink-antikink density wave for the sensitivity (the inverse of delay time) $a=3.5$. The density wave is robust. (b) The chaotic density waves for $a=5.0$. They coalesce with one another $(a \rightarrow b)$ and break up $(c \rightarrow d)$.

$(\gamma, a)$ where $\rho_{0}=0.2$. Regions I, II, and III indicate, respectively, the uniform flow with no jam, the jammed flow of the kink density wave, and the jammed flow of the chaotic density wave. Curve $a_{c}=3 /(1-2 \gamma)$ represents the critical line predicted by the linear stability analysis (see Sec. IV). The boundary line between the uniform flow and the density wave flow obtained from the simulation coincides with the critical line. When the passing constant $\gamma$ is larger than 0.1 , the chaotic density waves appear. For values of $\gamma$ smaller than 0.1 , the chaotic density waves do not appear. For $\gamma$ $<0.1$, the traffic behavior is similar to that of the single-lane traffic without passing, except that the critical point $a_{c}$ $[=3 /(1-2 \gamma)]$ increases with $\gamma[17]$. The boundary line between the kink and chaotic density waves is the line of $a$ $=3.75$ for $0.1 \leqslant \gamma \leqslant 0.4$. With increasing $\gamma$, region III, chaotic jams, expands to that of large $a$. Region II, kink jams, remains the same as that of $\gamma=0.1$.

Figure 4 shows the typical density profiles obtained after $t=20000$ for $\gamma=0.4$. The density profiles (a), (b), and (c)

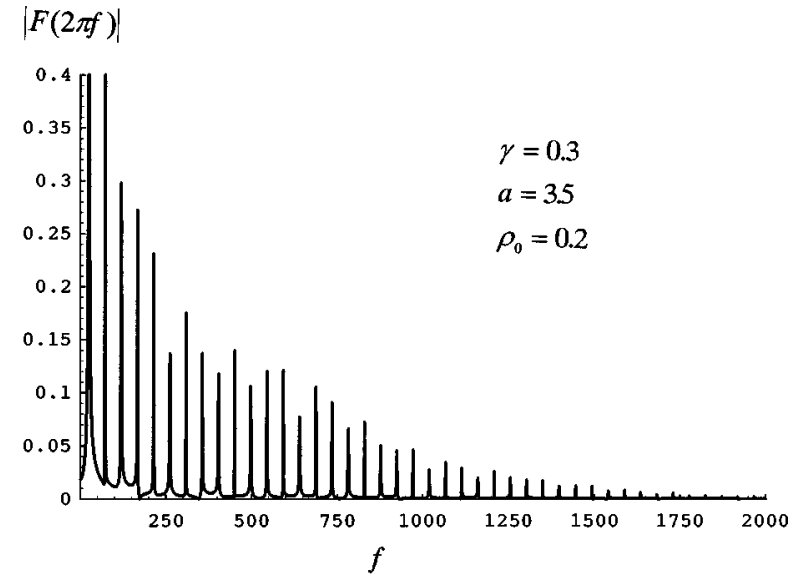

(a)

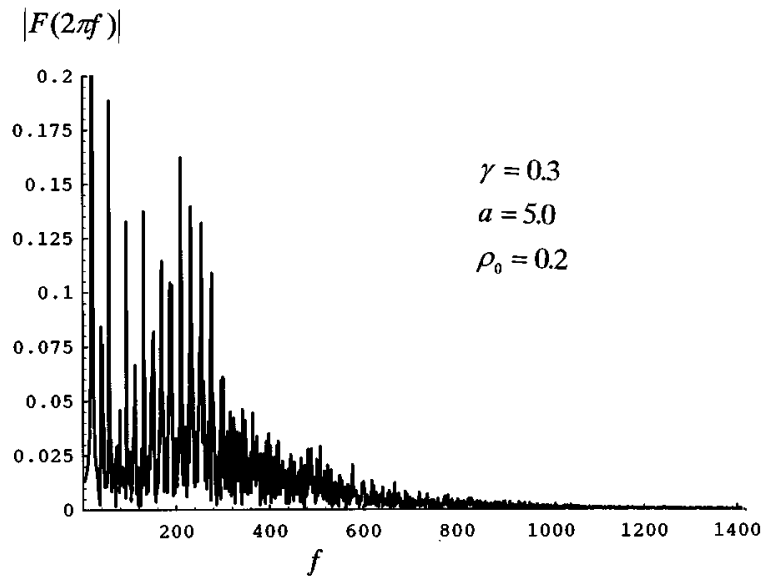

(b)

FIG. 2. Fourier spectra of time series of the density. They correspond to traffic patterns (a) and (b) in Fig. 1, respectively.

show the uniform traffic flow for $a=16.0$, the chaotic density wave flow for $a=3.75$, and the kink density wave flow for $a=3.5$, respectively, where $\gamma=0.4$ and $\rho_{0}=0.2$. The critical point is given by $a_{c}=15.0$ for $\gamma=0.4$. Profiles (a), (b), and (c) represent the typical density profiles of the traffic flows in regions I, III, and II, respectively. The traffic flow

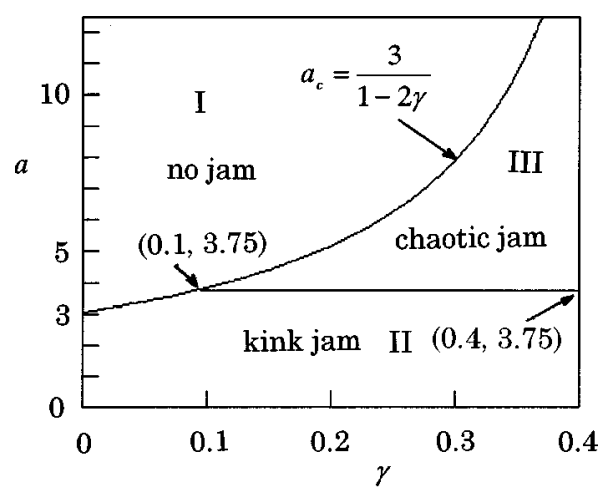

FIG. 3. Phase diagram in parameter space $(\gamma, a)$ where $\rho_{0}$ $=0.2$. Regions I, II, and III indicate, respectively, uniform flow with no jam, jammed flow of the kink density wave, and jammed flow of the chaotic density wave. 


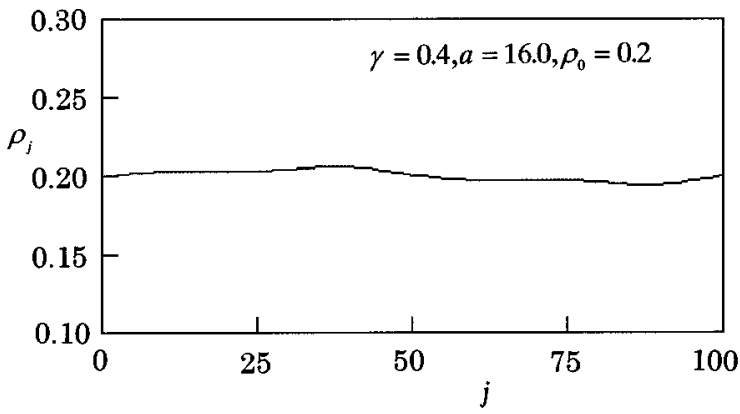

(a)

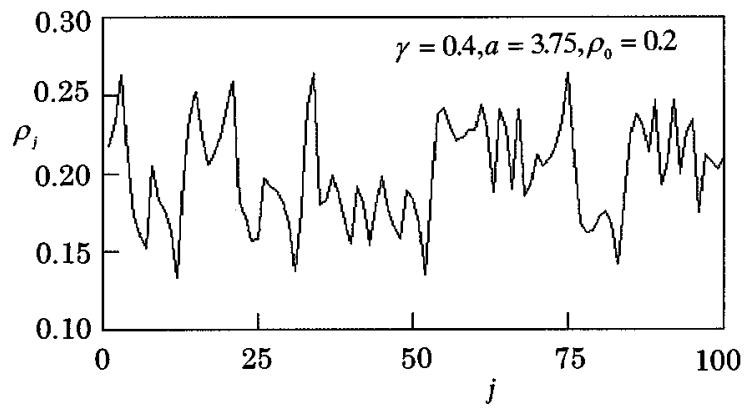

(b)

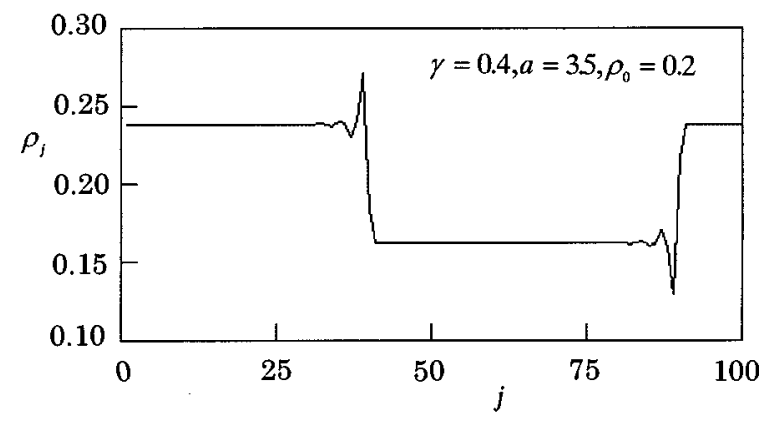

(c)

FIG. 4. Typical density profiles obtained after $t=20000$ for $\gamma$ $=0.4$. The density profiles (a), (b), and (c) exhibit, respectively, the uniform traffic flow for $a=16.0$, the chaotic density wave flow for $a=3.75$, and the kink density wave flow for $a=3.5$, where $\gamma$ $=0.4$ and $\rho_{0}=0.2$.

(a) obtained after a sufficiently long time is almost uniform spatially but fluctuates a little around $\rho_{0}=0.2$. When $\gamma$ $=0.0$, the fluctuation does not occur. The profile (b) exhibits the irregular pattern that is characteristic of chaos. The strength of density profile (b) increases as sensitivity $a$ (the inverse of delay time $\tau$ ) departs from critical point $a_{c}$ $=15.0$. The stronger chaotic jams appear as $a$ deviates from the critical point. The profile (c) exhibits the typical kinkantikink density wave. The kink density wave is similar to that observed in single-lane traffic [17], except for the spikes in the front and rear of the kink. When $\gamma=0.0$, the spikes do not appear.

Figure 5 shows the plots of density difference $\rho(t)-\rho(t$ -1 ) against density $\rho(t)$ for $t=20000-30000$, where $\rho(t)$ is the density at time $t$ at a position of the one-dimensional lattice. Plots (a), (b), and (c) correspond to the traffic flows (a), (b), and (c) in Fig. 4, respectively. When the density is uniform spatially, its uniform traffic is represented by a single point $\left(\rho_{0}, 0\right)$ in the phase-space plot, where $\rho_{0}=0.2$.

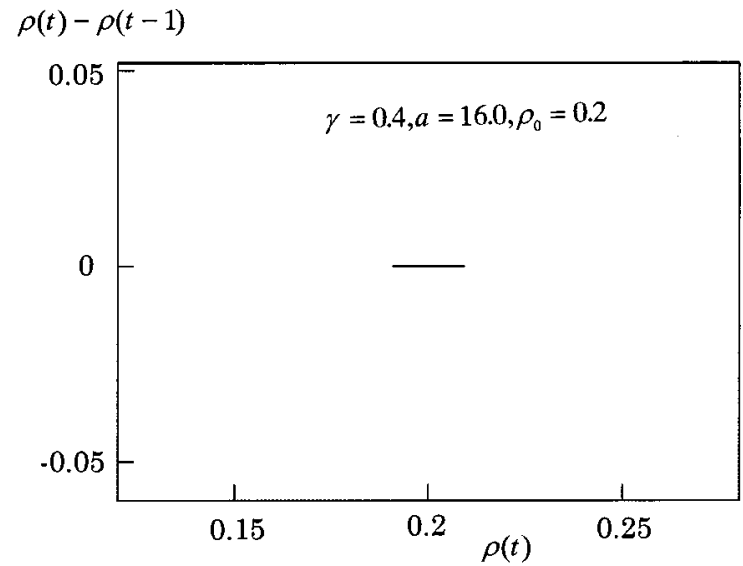

(a)

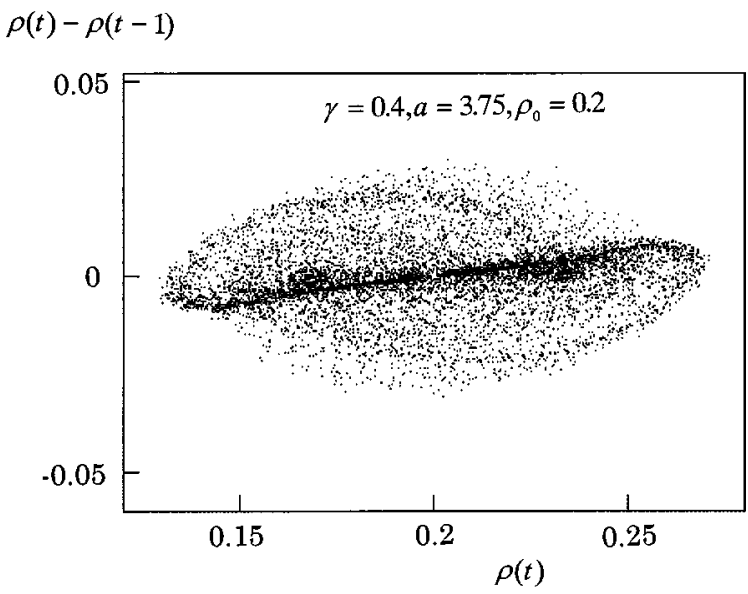

(b)

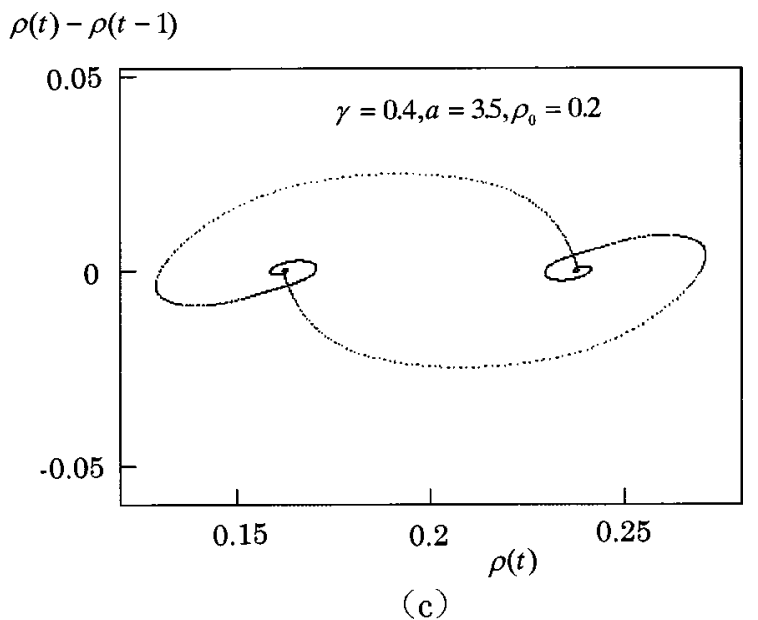

FIG. 5. Plots of the density difference $\rho(t)-\rho(t-1)$ against density $\rho(t)$ for $t=20000-30000$. Plots (a) -(c) correspond to traffic flows (a)-(c) in Fig. 4, respectively.

When $\gamma=0.0$, the uniform traffic flow is indeed plotted by the single point $(0.2,0.0)$. Plot (a) reflects the small and slow variation of density in Fig. 4(a). In this case, the traffic state is not represented by a single point, but is expressed by the bar in the phase-space plot. Plot (b) of the chaotic jam exhibits the behavior characteristic of the chaos. The chaotic traffic flow is represented by the set of dispersed points in the phase-space plot. This is characteristic of chaos. Plot (c) of 


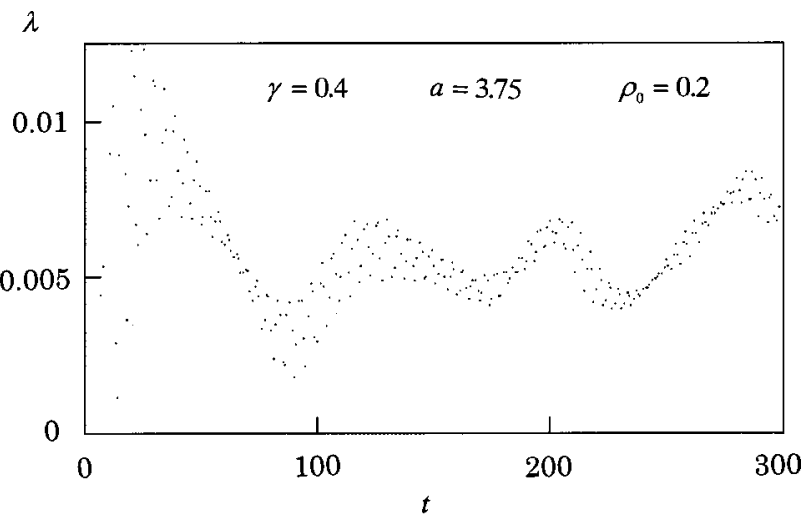

(a)

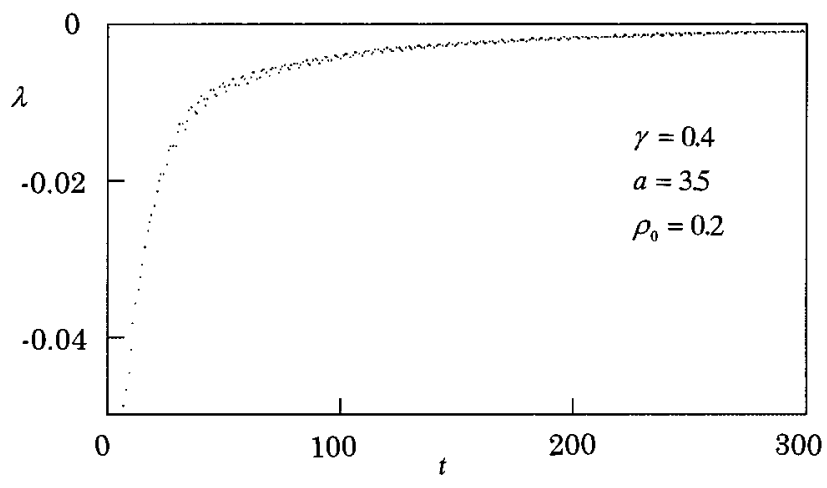

(b)

FIG. 6. Plots of Lyapunov exponent $\gamma$ against time $t$. (a) The Lyapunov exponent corresponding to the chaotic flow in Fig. 4(b). (b) The Lyapunov exponent corresponding to the kink density wave in Fig. 4(c).

the kink jam exhibits the limit cycle characteristic of the periodic pattern. The nodes on the right and left sides in plot (c) represent the traffic states within and out of the kink (traffic jam). When $\gamma=0.0$, the kink traffic flow is represented by an elliptical curve in the phase-space plot. Plot (c) reflects the kink-antikink jam, with the spikes shown in Fig. 4(c).

We calculate the Lyapunov exponent $\lambda$ of the time evolution of the density for the two density wave flows shown in Figs. 4 and 5. As a result, the chaotic traffic flow displays a positive Lyapunov exponent (a) in Fig. 6. This is characteristic of chaos. In contrast, the kink density wave flow displays a negative Lyapunov exponent (b) in Fig. 6. This is characteristic of regular motion. Thus, it is confirmed that the irregular jams within region III in Fig. 3 are in the chaotic state, by studying the phase-space plot, the Lyapunov exponent, and the Fourier spectrum. Indeed, the chaotic traffic flow bears a resemblance to dynamical chaos in classical mechanics [20]. Thus, we have justified the term "chaos" by computing Fourier spectra, phase-space plots, and Lyapunov exponents.

\section{LINEAR STABILITY ANALYSIS}

We apply the linear stability method to the traffic model described by Eq. (5). We consider the stability of the uniform traffic flow. The uniform traffic flow is defined by such a state as traffic flow with constant density $\rho_{0}$ and constant velocity $V\left(\rho_{0}\right)$. The solution of the uniform steady state is given by

$$
\rho_{j}(t)=\rho_{0}, \quad \mathrm{v}_{j}(t)=V\left(\rho_{0}\right) .
$$

Let $y_{j}(t)$ be a small deviation from the uniform steady-state flow: $\rho_{j}(t)=\rho_{0}+y_{j}(t)$. Then, the linear equation is obtained from Eq. (5),

$$
\begin{array}{r}
y_{j}(t+2 \tau)-y_{j}(t+\tau)+\tau \rho_{0}^{2} V^{\prime}\left[y_{j+1}(t)-y_{j}(t)\right] \\
-\gamma \tau \rho_{0}^{2} V^{\prime}\left[y_{j+2}(t)-2 y_{j+1}(t)+y_{j}(t)\right]=0,
\end{array}
$$

where $V^{\prime}=d V(\rho) /\left.d \rho\right|_{\rho=\rho_{0}}$.

By expanding $y_{j}(t) \propto \exp (i k j+z t)$, the following equation of $z$ is derived:

$$
e^{2 z \tau}-e^{z \tau}+\tau \rho_{0}^{2} V^{\prime}\left(e^{i k}-1\right)-\gamma \tau \rho_{0}^{2} V^{\prime}\left(e^{2 i k}-2 e^{i k}+1\right)=0 .
$$

By expanding $z=z_{1}(i k)+z_{2}(i k)^{2}+\cdots$, the first- and second-order terms of $i k$ are obtained

$$
z_{1}=-\rho_{0}^{2} V^{\prime}, \quad z_{2}=-\frac{3}{2} \tau\left(\rho_{0}^{2} V^{\prime}\right)^{2}-\frac{(1-2 \gamma)}{2} \rho_{0}^{2} V^{\prime}
$$

If $z_{2}$ is a negative value, the uniform steady-state flow becomes unstable for long-wavelength modes. When $z_{2}$ is a positive value, the uniform flow is stable. The neutral stability condition is given as

$$
\tau=-\frac{(1-2 \gamma)}{3 \rho_{0}^{2} V^{\prime}}
$$

For small disturbances of long wavelengths, the uniform traffic flow is unstable if

$$
\tau>-\frac{(1-2 \gamma)}{3 \rho_{0}^{2} V^{\prime}}
$$

The derivative $V^{\prime}\left(\rho_{0}\right)$ of the optimal velocity has the minimal value at turning point $\rho_{0}=\rho_{c}$. Therefore, if $\tau<\tau_{c}\left[\tau_{c}\right.$ $\left.=-(1-2 \gamma) / 3 \rho_{c}^{2} V^{\prime}=(1-2 \gamma) / 3\right]$, the uniform flow is always stable, irrespective of density. We find that there is a critical point at $\rho=\rho_{c}$ and $\tau=\tau_{c}$. When $\gamma=0$, the critical point and the neutral stability line agree with those in a single-lane traffic flow with no passing [17]. The solid curves in Fig. 7 show the neutral stability lines for $\gamma=0.0$, $0.1,0.2,0.3$, and 0.4 . The apex of each curve indicates the critical point. The traffic flow above each curve is stable and the traffic jam does not appear. Below each curve, the traffic flow is unstable and the density wave appears. The critical points and the neutral stability curves increase with passing constant $\gamma$. In the case of $\gamma=0.0$ without the passing, no traffic jams occur when sensitivity $a$ is larger than 3 . The traffic jams appear because of the passing effect, even if sensitivity $a$ is larger than 3 . This means that by introducing passing into the original single-lane traffic flow, the traffic flow becomes more unstable than that without no passing. The passing induces a more unstable traffic flow than traffic 


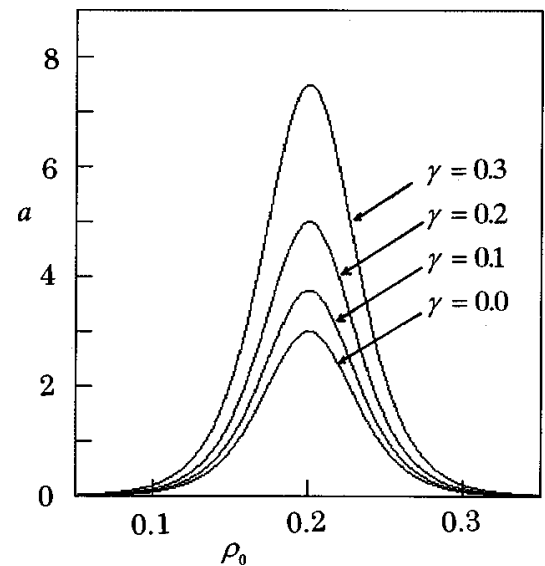

FIG. 7. Neutral stability lines obtained by the linear stability analysis for $\gamma=0.0,0.1,0.2,0.3$, and 0.4 . The apex of each curve indicates the critical point.

without the passing. With the increase of $\gamma$, the uniform flow becomes more unstable. Unfortunately, the chaotic traffic flow is not predicted by the linear stability theory.

\section{NONLINEAR ANALYSIS}

We now consider long-wavelength modes in the traffic flow on coarse-grained scales. The simplest way to describe the long-wavelength modes is the long-wave expansion. We consider the slowly varying behavior at long wavelengths near the critical point $\rho_{c}, \tau_{c}$. We extract slow scales for space variable $j$ and time variable $t[13,17,21]$. For $0<\varepsilon$ $\ll 1$, we therefore define slow variables $X$ and $T$.

$$
X=\varepsilon(j+b t), \quad T=\varepsilon^{3} t,
$$

where $b$ is a constant to be determined later. We set the density as

$$
\rho_{j}(t)=\rho_{c}+\varepsilon R(X, T) .
$$

By expanding Eq. (5) to the fifth order of $\varepsilon$ with the use of Eqs. (14) and (15), one obtains the following nonlinear partial-differential equation:

$$
\begin{aligned}
\varepsilon^{2}\left(b+\rho_{c}^{2} V^{\prime}\right) \partial_{X} R+\varepsilon^{3}\left(\frac{3 b^{2} \tau}{2}+\frac{(1-2 \gamma)}{2} \rho_{c}^{2} V^{\prime}\right) \partial_{X}^{2} R \\
+\varepsilon^{4}\left[\partial_{T} R+\left(\frac{7 b^{3} \tau^{2}}{6}+\frac{\rho_{c}^{2} V^{\prime}}{6}-\gamma \rho_{c}^{2} V^{\prime}\right) \partial_{X}^{3} R\right. \\
\left.+\frac{\rho_{c}^{2} V^{\prime \prime \prime}}{6} \partial_{X} R^{3}\right]+\varepsilon^{5}\left[3 b \tau \partial_{X} \partial_{T} R+\left(\frac{5 b^{4} \tau^{3}}{8}+\frac{\rho_{c}^{2} V^{\prime}}{24}\right.\right. \\
\left.\left.-\frac{7 \gamma \rho_{c}^{2} V^{\prime}}{12}\right) \partial_{X}^{4} R+\frac{\rho_{c}^{2} V^{\prime \prime \prime}}{12} \partial_{X}^{2} R^{3}-\frac{\gamma \rho_{c}^{2} V^{\prime}}{2} R^{2} \partial_{X}^{2} R\right]=0,
\end{aligned}
$$

where $\quad V^{\prime}=d V(\rho) /\left.d \rho\right|_{\rho=\rho_{c}}$ and $V^{\prime \prime \prime}=d^{3} V(\rho) /\left.d \rho^{3}\right|_{\rho=\rho_{c}}$. Here we used the expansions shown in the Appendix.

By taking $b=-\rho_{c}^{2} V^{\prime}$, the second-order term of $\varepsilon$ is eliminated from Eq. (16). We consider the neighborhood of the critical point $\tau_{c}$ :

$$
\frac{\tau}{\tau_{c}}=1+\varepsilon^{2},
$$

where $\tau_{c}=-(1-2 \gamma) / 3 \rho_{c}^{2} V^{\prime}=(1-2 \gamma) / 3$. Equation (16) is rewritten as

$$
\begin{aligned}
& \varepsilon^{4}\left(\partial_{T} R-\frac{\left(1-13 \gamma-14 \gamma^{2}\right)}{27}\left(-\rho_{c}^{2} V^{\prime}\right) \partial_{X}^{3} R+\frac{\rho_{c}^{2} V^{\prime \prime \prime}}{6} \partial_{X} R^{3}\right) \\
& +\varepsilon^{5}\left(\frac{(1-2 \gamma)}{2}\left(-\rho_{c}^{2} V^{\prime}\right) \partial_{X}^{2} R+\frac{\left(1-6 \gamma+39 \gamma^{2}+46 \gamma^{3}\right)}{54}\right. \\
& \quad \times\left(-\rho_{c}^{2} V^{\prime}\right) \partial_{X}^{4} R-\frac{(1-4 \gamma)}{12} \rho_{c}^{2} V^{\prime \prime \prime} \partial_{X}^{2} R^{3} \\
& \left.\quad-\frac{\gamma}{2} \rho_{c}^{2} V^{\prime \prime \prime} R^{2} \partial_{X}^{2} R\right)=0 .
\end{aligned}
$$

In order to derive the regularized equation, we make the following transformation for Eq. (18):

$$
\begin{aligned}
T^{\prime} & =\frac{\left(1-13 \gamma-14 \gamma^{2}\right)\left(-\rho_{c}^{2} V^{\prime}\right)}{27} T, \\
R & =\left(-\frac{2\left(1-13 \gamma-14 \gamma^{2}\right) \rho_{c}^{2} V^{\prime}}{9 \rho_{c}^{2} V^{\prime \prime \prime}}\right)^{1 / 2} R^{\prime},
\end{aligned}
$$

where we assumed

$$
1-13 \gamma-14 \gamma^{2}>0 .
$$

One obtains the regularized equation

$$
\begin{aligned}
\partial_{T^{\prime}} R^{\prime}= & \partial_{X}^{3} R^{\prime}-\partial_{X} R^{\prime 3}-\varepsilon\left(\frac{27(1-2 \gamma)}{2\left(1-13 \gamma-14 \gamma^{2}\right)} \partial_{X}^{2} R^{\prime}\right. \\
& +\frac{\left(1-6 \gamma+39 \gamma^{2}+46 \gamma^{3}\right)}{2\left(1-13 \gamma-14 \gamma^{2}\right)} \partial_{X}^{4} R^{\prime} \\
& \left.-\frac{(1-4 \gamma)}{2} \partial_{X}^{2} R^{\prime 3}-3 \gamma R^{\prime 2} \partial_{X}^{2} R^{\prime}\right) .
\end{aligned}
$$

If we ignore the $O(\varepsilon)$ terms in Eq. (21), this is just the modified $\mathrm{KdV}$ equation with a kink solution as the desired solution,

$$
R_{0}^{\prime}\left(X, T^{\prime}\right)=\sqrt{c} \tanh \sqrt{\frac{c}{2}}\left(X-c T^{\prime}\right)
$$

The selected value of propagation velocity $c$ for the kink solution is determined from the $O(\varepsilon)$ term. The kink solution (22) is obtained only when the condition (20) is satisfied. The kink solution exists only for

$$
0 \leqslant \gamma<\frac{1}{14} .
$$

Thus, we have proven that the modified $\mathrm{KdV}$ equation with a kink solution is derived only for $0 \leqslant \gamma<\frac{1}{14}$. For values of $\gamma$ larger than $\frac{1}{14}$, the modified KdV equation cannot be derived from the above nonlinear analysis. The simulation result in Fig. 3 exhibits the chaotic jam for $\gamma>0.1$. The nonlinear 
analysis result is not inconsistent with the simulation result. The kink density wave can be described by the modified $\mathrm{KdV}$ equation, but the chaotic density waves cannot be explained by the nonlinear analysis. This may be due to the singular point at $\gamma=\frac{1}{14}$. When $\gamma$ is larger than $\frac{1}{14}$, the scaling assumption (14) breaks down. Therefore, a scaling assumption that is different from Eq. (14) will be necessary to derive the nonlinear wave equation describing the chaotic density wave.

\section{SUMMARY}

We have proposed a lattice hydrodynamic model that takes into account the passing effect in the traffic flow on a highway. We have investigated the traffic behavior by computer simulation, the linear stability analysis, and the nonlinear analysis. We have found that the chaotic jams appear when the passing constant is larger than 0.1 . The chaotic traffic has been investigated by computing the Fourier spectrum, phase-space plot, and Lyapunov exponent. We have shown that the transition line between the uniform traffic and density wave flows coincides with the neutral stability line based on the linear stability theory. We have proven that the modified $\mathrm{KdV}$ equation can be derived for small values of the passing constant by use of the nonlinear analysis. We have found that passing has an important effect on the traffic flow.

To our knowledge, this paper is the first work to show that chaotic jams occur by taking passing into account in the deterministic traffic model. It is expected, as a next step, that one will derive the nonlinear differential equation describing the traffic chaos.

\section{APPENDIX}

In this appendix, we give the expansions of each term in Eq. (5) to fifth order of $\varepsilon$ :

$$
\begin{aligned}
\rho_{j}(t+\tau)= & \rho_{c}+\varepsilon R+\varepsilon^{2} b \tau \partial_{X} R+\varepsilon^{3} \frac{(b \tau)^{2}}{2} \partial_{X}^{2} R \\
& +\varepsilon \frac{(b \tau)^{3}}{6} \partial_{X}^{3} R+\varepsilon^{4} \tau \partial_{T} R \\
& +\varepsilon^{5} \frac{(b \tau)^{4}}{24} \partial_{X}^{4} R+\varepsilon^{5} b \tau^{2} \partial_{T} \partial_{X} R \\
\rho_{j}(t+2 \tau)= & \rho_{c}+\varepsilon R+\varepsilon^{2} 2 b \tau \partial_{X} R+\varepsilon^{3} \frac{(2 b \tau)^{2}}{2} \partial_{X}^{2} R \\
& +\varepsilon^{4} \frac{(2 b \tau)^{3}}{6} \partial_{X}^{3} R+\varepsilon^{4} 2 \tau \partial_{T} R \\
& +\varepsilon^{5} \frac{(2 b \tau)^{4}}{24} \partial_{X}^{4} R+\varepsilon^{5} 4 b \tau^{2} \partial_{T} \partial_{X} R \\
& +\frac{\varepsilon^{4} 8}{6} \partial_{X}^{3} R+\frac{\varepsilon^{5} 16}{24} \partial_{X}^{4} R \\
\rho_{j+2}(t)= & \rho_{c}+\varepsilon R+\varepsilon^{2} 2 \partial_{X} R+\frac{\varepsilon^{3} 4}{2} \partial_{X}^{2} R \\
& +\varepsilon^{2} \partial_{X} R+\frac{\varepsilon^{3}}{2} \partial_{X}^{2} R+\frac{\varepsilon^{4}}{6} \partial_{X}^{3} R+\frac{\varepsilon^{5}}{24} \partial_{X}^{4} R
\end{aligned}
$$

We expand the optimal velocity function at the turning point:

$$
V\left(\rho_{j}\right)=V\left(\rho_{c}\right)+V^{\prime}\left(\rho_{c}\right)\left(\rho_{j}-\rho_{c}\right)+\frac{V^{\prime \prime \prime}\left(\rho_{c}\right)}{6}\left(\rho_{j}-\rho_{c}\right)^{3} \text {. }
$$

By inserting (A1)-(A5) into Eq. (5), one obtains Eq. (16).
[1] Traffic and Granular Flow, edited by D. E. Wolf, M. Schreckenberg, and A. Bachem (World Scientific, Singapore, 1996).

[2] D. Helbing, Verkehrsdynamik (Springer, Berlin, 1997).

[3] M. Bando, K. Hasebe, A. Nakayama, A. Shibata, and Y. Sugiyama, Phys. Rev. E 51, 1035 (1995).

[4] K. Nagel and M. Schreckenberg, J. Phys. I 2, 2221 (1992).

[5] E. Ben-Naim, P. L. Krapivsky, and S. Redner, Phys. Rev. E 50, 822 (1994).

[6] D. Helbing, Phys. Rev. E 53, 2366 (1996).

[7] O. Biham, A. A. Middleton, and D. A. Levine, Phys. Rev. A 46, R6124 (1992).

[8] T. Nagatani, Phys. Rev. E 48, 3290 (1993).

[9] J. A. Cuesta, F. C. Martinez, J. M. Nolera, and A. Sanchez, Phys. Rev. E 48, 4175 (1993).

[10] B. S. Kerner, P. Konhauser, and M. Schilke, Phys. Rev. E 51, 6243 (1995).
[11] S. Krauss, P. Wagner, and C. Gawron, Phys. Rev. E 55, 5597 (1997).

[12] B. S. Kerner and H. Rehborn, Phys. Rev. E 53, R1297 (1996).

[13] T. Komatsu and S. Sasa, Phys. Rev. E 52, 5574 (1995).

[14] T. Nagatani and K. Nakanishi, Phys. Rev. E 57, 6415 (1998).

[15] T. Nagatani, K. Nakanishi, and H. Emmerich, J. Phys. A 31, 5431 (1998).

[16] T. Nagatani, Phys. Rev. E 58, 4271 (1998).

[17] T. Nagatani, Physica A 264, 581 (1999).

[18] G. F. Newell, Oper. Res. 9, 209 (1961).

[19] G. B. Whitham, Proc. R. Soc. London, Ser. A 428, 49 (1990).

[20] H. G. Schuster, Deterministic Chaos (VCH, Weinheim, 1988).

[21] M. C. Cross and P. C. Hohenberg, Rev. Mod. Phys. 65, 851 (1993). 